(HPV) has been identified as the causal agent of cervical cancer. Project 'HOPE Peru: Women helping women fight cervical cancer' aims to create the first Peruvian public health social enterprise aiming to improve access to healthcare technologies for communities with the involvement of community women. HOPE's first project will be to seek to market the HPV selftesting $\left(\mathrm{CareHPV}^{\circledR}\right)$ to get commitment and promote a culture of cervical cancer prevention. The tests will be sold to high income women to create a sustainable platform to offer free testing to women with less resources, involving training of community women ('HOPE ladies'). The project is based in four key pillars: (1) the use of molecular HPV tests for screening, with better sensitivity than PAP tests and at a relatively low cost; (2) the use of self-collected vaginal samples, which offers an opportunity to increase screening coverage; (3) community women teaching other women about cervical cancer and how to apply the HPV test; and (4) use of technology with the development of an informatics platform for the follow up of the distribution of molecular HPV screening tests, results, follow-up of women screened and the transmission of reminders through text messages (SMS) for clinic visits to women and an internet information platform and hot-line. The HPV test can be self-administered by women in the comfort and privacy of their own homes. Depending on the case the test could be pick-up from their houses or could be deposited in collection boxes located in commercial places (pharmacies, stores) opened 24/7. The samples are tested at a central lab and the results of the test are received within a week via SMS, with appropriate referrals for treatment as needed.

Disclosure No significant relationships.

\section{SO2.4 EMPOWERMENT AND APPROACHES FOR STIGMA REDUCTION: IMPLEMENTATION OF HIV SELF-TESTING AMONG FEMALE SEX WORKERS}

Catherine Oldenburg*. University of California, San Francisco, USA

10.1136/sextrans-2019-sti.23

HIV self-testing (HIVST) may play a role in addressing gaps in HIV testing coverage and as an entry point for HIV prevention services by empowering individuals to test themselves and reducing stigma-related barriers to HIV testing. We evaluated two health systems delivery approaches for HIVST distribution compared to referral to standard testing among female sex workers in Zambia. Trained peer educators in Kapiri, Chirundu, and Livingstone, Zambia each recruited 6 FSW participants. Peer educator-FSW groups were randomized to one of three arms: 1) delivery (direct distribution of an oral HIVST from the peer educator), 2) coupon (a coupon for collection of an oral HIVST from a health clinic/pharmacy), or 3) standard-of-care HIV testing. Participants in the two HIVST arms received two kits: one at baseline and one at three months. The primary outcome was any self-reported HIV testing in the past month at the one- and four-month visits, as HIV self-testing can replace other parts of HIV testing. Secondary endpoints included linkage to care, HIVST use in the intervention arms, adverse events, empowerment, sexual behaviors, and measures of stigma. Participants completed questionnaires at one and four months following peer educator interventions.965 participants were enrolled (delivery: $\mathrm{N}=322$, coupon, $\mathrm{N}=323$, standard, $\mathrm{N}=320$ ); $20 \%$ had never tested for HIV. Overall HIV testing at one month was 94.9\% in the delivery arm, $84.4 \%$ coupon, and $88.5 \%$ standard-ofcare. Four month rates were $84.1 \%$ delivery, $79.8 \%$ coupon and $75.1 \%$ standard. HIV self-test use was higher in the delivery arm compared to the coupon arm $(\mathrm{RR}=1.14$, 95\% CI $1.05-1.23, P=0.001)$ at one month but there was no difference in at four months. Among participants reporting a positive HIV test at one $(\mathrm{N}=144)$ and four months $(\mathrm{N}=235)$, linkage to care was non-significantly lower in the two HIVST arms compared to the standard-of-care arms. At four months, participants reported significantly fewer clients per night in the delivery arm (mean difference -0.78 clients, 95\% CI -1.28 to $-0.28, P=0.002)$ and the coupon arm $(-0.71,95 \%$ CI -1.21 to $-0.21, P=0.005)$ compared to standard-of-care. HIV selftesting coverage was high in all arms, suggesting that HIV self-testing is able to overcome stigma-related barriers to HIV testing in this population.

Disclosure No significant relationships.

\section{S03 - EPIDEMIOLOGICAL ASPECTS OF STI TRANSMISSION IN MSM}

\section{Monday, July 15, 2019 10:45 AM - 12:15 PM}

\section{S03.1 IMPACT OF HIV PREP ON RISK COMPENSATION AND STI EPIDEMIOLOGY - WHAT DOES THE EVIDENCE SHOW?}

Helen Ward*. Imperial College London, London, UK

\subsection{6/sextrans-2019-sti.24}

Introduction From the earliest days of the HIV epidemic there has been a close relationship with other sexually transmitted infections (STI). The shared transmission routes and determinants were reflected in high levels of synergy in the epidemics. Early preventive interventions for HIV - including changes in partner numbers and selection, use of barriers and changes in sexual practices towards safer sex - were 'infection agnostic', and had a dramatic impact on bacterial STI, with levels of syphilis and gonorrhoea falling to historic lows in some high-income settings. In contrast, many newer technologies for HIV prevention are 'infection specific', leading to the potential for divergent epidemics of HIV and other STI

Method and results We review evidence to date of the impact of PrEP on (a) risk compensation, and (b) STI rates in a range of populations and settings. We synthesize data from earlier systematic reviews, and review the association between PrEP use and bacterial STIs in cis-gender women. Detailed results will be presented; briefly, early randomised control trials reported no increase in STIs or changes in sexual practices; more recent studies in less controlled environments such as open-label or demonstration projects have often reported increased STI incidence and risk compensation. The majority of evidence is from studies in men who have sex with men and transgender women

Discussion An increase in condomless sex is not an unintended consequence of PrEP. For decades HIV prevention was limited because many people prefer sex without condoms. Now we have the technology to do this without the fear of HIV, just 
as the oral contraceptive pill (OCP) removed the fear of pregnancy for many women. While that may contribute to an increase in STI, as the OCP did, we should use this as an opportunity to argue for renewed investment in STI control programmes and research.

Disclosure No significant relationships.

\section{SO3.2 THE ROLE OF ORAL-ANAL TRANSMISSION IN PERSISTENCE OF CHLAMYDIAL INFECTION}

Henry De Vries*. Amsterdam University Medical Centres, Dermatology, Amsterdam, Netherlands

\subsection{6/sextrans-2019-sti.25}

In virtually every mammal, chlamydiae strains persist in the gastro-intestinal tract for long periods of time in the absence of apparent inflammation and pathology. Although anorectal Chlamydia trachomatis (CT) infections are diagnosed frequently in women and men who have sex with men, their detection often remains unexplained as anal sex is not always reported. Oropharyngeal infections and the gastro-intestinal tract may contribute to the explanation. Both symptomatic and asymptomatic chlamydial pharyngitis has been described. Consequentially, the pathogen may pass through the gastrointestinal tract to the large intestine and rectum, inducing chlamydia proctitis, and ongoing transmission. Oro-anal transmission might also explain the asymmetric distribution of diagnosed anorectal and urogenital lymphogranuloma venereum infections among men who have sex with men as found in the current epidemic. Recently the use of saliva as lubricant for anal sex has been proposed as alternative (oro-anal) route for gonorrhoea transmission in MSM. It can be deduced that this mode of transmission further contributes to the exceeding numbers of anorectal LGV infections as opposed to genital infections.

Disclosure No significant relationships.

\section{S03.3 SEXUAL TRANSMISSION OF N. MENINGITIDIS IN MSM AND IMPLICATIONS FOR PUBLIC HEALTH}

Anna McNulty*. Sydney Sexual Health Centre, Sydney, Australia

\subsection{6/sextrans-2019-sti.26}

Over the last 5 years we have seen outbreaks of N. meningitidis urethritis and invasive meningococcal disease (IMD) in gay and bisexual men in various centres around the world with speculation as to what has driven these outbreaks. As a result there has been a significant focus on the genomics of the isolates associated with the recent outbreaks and in particular horizontal gene transfer from $N$. gonorrhoeae and the impact on the virulence of $N$. meningitidis Vaccination against meningococcus has impacted the epidemiology of this infection broadly however the impact in MSM is less clear. There is relatively little recent data about the prevalence of asymptomatic carriage in MSM particularly in the era of PrEP. Commonly, STI management guidelines do not include sexually transmitted $N$. meningitidis and there are varying laboratory practices with regard to notification of isolates and public health guidance is variable as well. In this presentation the epidemiology of N. meningitidis in MSM around the world will be explored. The potential drivers of these outbreaks will be examined and in particular the genomic investigations into clonal complex 11. The public health responses to date and the implications for public health as well as STI clinicians will be examined.

Disclosure No significant relationships.

\section{SO3.4 HEPATITIS C IN HIV-NEGATIVE MSM - A GROWING CONCERN?}

Axel Schmidt*. London School of Hygiene and Tropical Medicine/SIGMA Research, London, UK

\subsection{6/sextrans-2019-sti.27}

In post-industrialised countries, since the turn of the millennium we have witnessed outbreaks of hepatitis C among HIVdiagnosed gay men, and cohort studies of HIV-diagnosed men-who-have-sex-with-men (MSM) have shown high incidence of HCV infection, or re-infection after clearance. Clinicians and health promotion specialists have repeatedly expressed concerns that the HCV epidemic will jump to HIVnegative MSM. For the last two decades there has been a widespread perception that HCV will inevitably affect the whole population of MSM. For many years, studies of HIVnegative MSM have provided no strong evidence for an HCV sero-prevalence higher than among other adult men, and no outbreaks had been observed among HIV-negative MSM. It has hence been conceptualised that HIV infection itself might be a major risk factor for HCV acquisition, due to alterations of cellular immunity, but the risk attributable to immune dysfunction is still unclear. Some have argued that sero-adaptive sexual networks might better explain the observed differences between HIV-diagnosed and other MSM. As for behavioural factors, while the role of condomless anal sex for HCV transmission is controversial, there is consensus that mucosal disruption and sexual exposure to blood are major risk factors. With the recent advent of oral HIV pre-exposure prophylaxis, shifts in sexual networks have been observed as well as rising $\mathrm{HCV}$ incidence in PrEP-using HIV-negative MSM. This reignites previous fears about the imminence of an HCV epidemic among HIV-negative MSM. While intravenous drug use with shared equipment has been known for decades to be a leading risk factor for HCV transmission -regardless of HIV infection or sexual orientation- its impact on HCV transmission in MSM is understudied. This talk aims at (1) summarising the available literature on HCV transmission, focussing on HIVnegative MSM, and (2) providing a conceptual framework of sexual and sex-associated HCV transmission in gay men.

Disclosure No significant relationships. 